\title{
Vibrational Assignments of Acetyliodide and Deuterated Species, based on Infrared Gas Spectra and Valence Force Field
} Calculations

\author{
FLEMMING M. NICOLAISEN and J. STOKKEBRO HANSEN
}

University of Copenhagen, Chemical Laboratory V, The H. C. Ørsted Institute, Universitetsparken 5, DK-2100 Copenhagen, Denmark

The infrared gas phase spectra $\left(4000-50 \mathrm{~cm}^{-1}\right)$ of acetyliodide (AcI), AcI- $d_{1}$, AcI- $d_{2}$ and AcI- $d_{3}$ are reported. A complete vibrational assignment is given, together with a valence force field calculation. The quality of the frequency refinement is shown to be sensitive to the methyl group structure. Minor corrections to the earlier methyl group structure are suggested.

The occurrence of acetyliodide (AcI) in the spectroscopic literature is rather sparse. A Raman investigation on liquid AcI was reported in $1948,{ }^{1}$ and an infrared investigation on liquid and gaseous AcI was reported in $1968 .{ }^{2}$ Recently McKean $^{3}$ has reported the $\mathrm{CH}$-stretching frequencies for $\mathrm{AcI}$ and $\mathrm{AcI}-d_{2}$. In the latter work the deviation from $C_{3 v}$ symmetry of the methyl group is discussed. The structure of gaseous AcI was determined from microwave spectra ${ }^{4}$ and from electron diffraction, ${ }^{5}$ the latter reporting a tilt of the methyl group of $1.7 \pm 5.4^{\circ}$. In the present work the infrared gas spectra of AcI, AcI- $d_{1}$, AcI- $d_{2}$ and AcI- $d_{3}$ are reported in the region $4000-50 \mathrm{~cm}^{-1}$. A complete vibrational assignment is given, which is in part based on a valence force field calculation. The symmetry co-ordinates used in the calculation allow deviation from $C_{3 v}$ symmetry for the methyl group. The frequency fit appeared to be sensitive to the methyl group structure, and a slightly revised methyl group structure is suggested.

\section{EXPERIMENTAL}

AcI was synthesized from ketene and HI. Ketene was prepared by pyrolysis of acetic acid anhydride $\left(600^{\circ} \mathrm{C}\right) .{ }^{6} \mathrm{HI}$ was prepared by adding $\mathrm{H}_{2} \mathrm{O}$ to $\mathrm{PI}_{3}$. Deuterated ketene and DI were made by a similar procedure using deuterated compounds. The isotopic purity (atomic percent) of ketene- $d_{2}$ was $99 \%$ and of DI $c a .90 \%$. AcI- $d_{3}$, AcI- $d_{2}$ and AcI- $d_{1}$ were prepared from ketene- $d_{2}$ and DI, ketene- $d_{2}$ and HI, and from ketene and DI respectively, the obtained isotopic purity being 90,99 and $90 \%$. The syntheses were carried out in a glass system under high vacuum. Excess of ketene or HI was removed by distillation. All contact with water from walls etc. must be avoided due to the rapid hydrolysis of AcI to

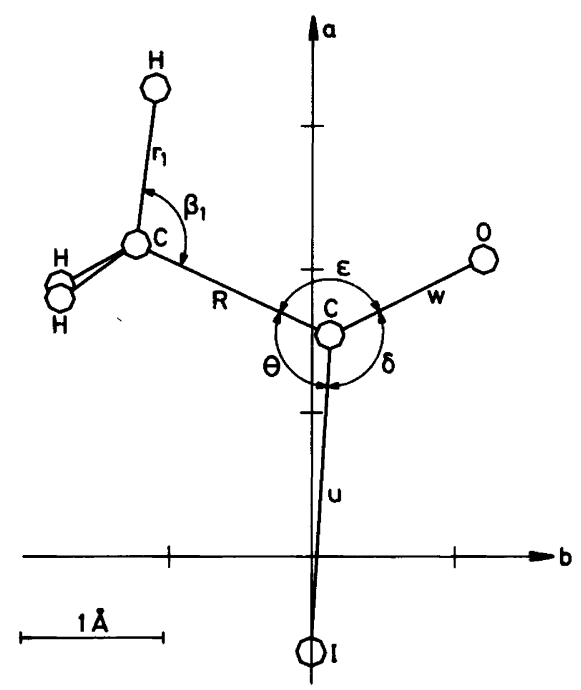

Fig. 1. Acetyliodide in the principal inertial axis system. 
acetic acid. Due to their variable relative intensity it was also possible to identify some weak lines in the IR-spectra caused by an unknown impurity (see Fig. 2).

Infrared gas spectra in the region 4000-350 $\mathrm{cm}^{-1}$ were recorded, using a $10 \mathrm{~cm}$ gas cell with $\mathrm{KBr}$ windows, on a Perkin-Elmer Model 580. The spectra were automatically stored in digitalized form in a medium size computer (RC 4000). This allowed computational removal of spectral interference from AcI in AcI- $d_{1}$, and from AcI- $d_{2}$ in AcI- $d_{3}$.

In the FIR-region $500-50 \mathrm{~cm}^{-1}$ a Michelson interferometer (RIIC, FS-520), equipped with a $4.6 \mathrm{~m}$ gas cell of the light pipe type, ${ }^{,}$was used.

For all the reported spectra a spectral resolution of $c a .2 \mathrm{~cm}^{-1}$ was used.

\section{RESULTS AND VIBRATIONAL ASSIGNMENT}

Acetyliodide is an asymmetric top molecule with a mirror plane as the only symmetry element (point group $C_{s}$ ). In Fig. $1 \mathrm{AcI}$ is shown in the principal axis system for the moments of inertia. The structure used is from Ref. 5. (See also Table 9.)

From symmetry arguments ${ }^{8}$ all fundamental frequencies can be expected to appear in the IR spectra, with ten bands of $A^{\prime}$-symmetry $(A / B$ hybrids) and five bands of $A^{\prime \prime}$-symmetry ( $C$-type bands). According to Ref. 9 the $C$-type bands will show a dominant $Q$-branch, the $B$-type bands a band minimum and the $A$-type bands a distinct but weak $Q$-branch. The $P, R$-separation is calcu- lated ${ }^{10,11}$ to be 12.6 and $10.5 \mathrm{~cm}^{-1}$ for the $A$ - and $B$-type bands, respectively. The changes in moments of inertia due to isotopic substitution are small and have no significant influence on calculated band contours. The experimental band contours are in good agreement with the calculated values.

For AcI- $d_{1}$ and AcI- $d_{2}$ two rotamers exist. One of $C_{s}$ symmetry and two equivalent forms with no symmetry $\left(C_{1}\right)$. The species of $C_{1}$ symmetry is expected to be twice as abundant as the species of $C_{s}$ symmetry. In general all absorption bands of the $C_{1}$ species will be $A / B / C$-hybrids.

In Tables 1-4 the observed band center frequencies are given together with relative intensities, band contours and vibrational assignment. In general the vibrational assignment for the parent molecule and the $d_{3}$-species is based on group frequencies and band contours.

$\mathrm{CH}$ - and $\mathrm{CD}$-stretching frequencies. In Fig. 2 the infrared spectrum of saturated vapours of AcI in a $10 \mathrm{~cm}$ cell is shown. The most striking feature is the relatively low intensity of the $\mathrm{CH}$-stretching bands near $3000 \mathrm{~cm}^{-1}$. The relative intensity of the CH-stretching modes for the isotopic compounds are qualitatively as seen in Fig. 2. The CD-stretching bands near $2100-2300 \mathrm{~cm}^{-1}$, which theoretically have half the intensity of the CH-stretching bands, mix with several weak overtone bands in this region, preventing a rigorous assignment of the $\mathrm{CD}$-stretching fundamentals. A tentative assignment is given in Tables 2-4.

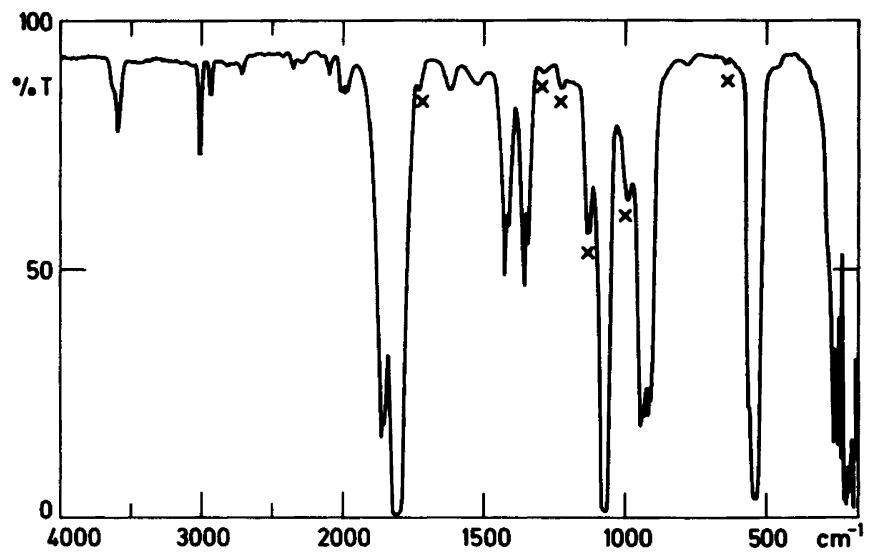

Fig. 2. Survey gas spectrum of acetyliodide (saturated vapours in a $10 \mathrm{~cm}$ cell). The bands marked with a cross are due to an unidentified impurity. 
Table 1. Tables 1-4 give the observed infrared gas phase data for AcI, AcI- $d_{3}$, AcI- $d_{1}$ and AcI- $d_{2}$. Given for each band is the observed frequency, the band contour, the relative intensity and a vibrational assignment.

For the $A$ - and the $C$-type bands the frequency corresponds to the value of the $Q$-branch maximum, and for the $B$-type bands to the central band minimum. In the spectrum of AcI- $d_{2}$ it can be seen that a band frequently has two closely separated $Q$-branches of nearly equal intensity. Such $Q$-branches are marked $C^{\prime}$ and $C^{\prime \prime}$. In the calculations the average frequency is used. The relative intensities are given by: $s=$ strong, $\mathrm{m}=$ medium, $\mathbf{w}=$ weak, vs=very strong etc. $\mathrm{F}$, Fermi resonance; sh, shoulder; ${ }^{*}$, diffuse band contour; +, overlapping bands.

Infrared gas phase data and vibrational assignment for acetyliodide.

\begin{tabular}{|c|c|c|c|}
\hline $\begin{array}{l}\text { Frequency } \\
\left(\mathrm{cm}^{-1}\right)\end{array}$ & $\begin{array}{l}\text { Band } \\
\text { contour }\end{array}$ & $\begin{array}{l}\text { Inten- } \\
\text { sity }\end{array}$ & $\begin{array}{l}\text { Assign- } \\
\text { ment }\end{array}$ \\
\hline 3595 & $B$ & vw & $2 v_{3}(3634)$ \\
\hline 3014 & $A$ & $\mathbf{w}$ & $v_{1}$ \\
\hline 2937 & $A$ & vw & $v_{2}$ \\
\hline 2814 & $\star$ & vw & $2 v_{4}(2842)$ \\
\hline 2717 & $B$ & vw & $v_{3}+v_{7}(2745)$ \\
\hline ca. 2360 & * & vw & $v_{3}+v_{8}(2368)$ \\
\hline ca. 2290 & $B$ & vw & $v_{5}+v_{7}(2281)$ \\
\hline 2104 & $*$ & vw & $v_{3}+v_{9}(2108)$ \\
\hline $\int 2000$ & $\boldsymbol{B}$ & vw & $v_{6}+v_{7}(2020)$ \\
\hline$+\{1993$ & $B$ & vw & $v_{6}+2 v_{14}(1993)$ \\
\hline 1860 & $B$ & $\mathbf{s}$ & $\left.2 v_{7}(1856)\right\} \mathrm{F}$ \\
\hline 1811 & $B$ & vs & $\left.v_{3}\right\} \mathrm{F}$ \\
\hline $\int 1646$ & $*$ & vw & $v_{5}+v_{9}(1644)$ \\
\hline$\{1624$ & $A$ & vw & $v_{6}+v_{8}(1630)$ \\
\hline $\int 1429$ & $C$ & $\mathrm{~m}$ & $v_{12}$ \\
\hline$+\{1421$ & $A / B$ & $\mathbf{m}$ & $v_{4}$ \\
\hline 1408 & $C$ & $\mathbf{w}$ & $v_{7}+v_{14}(1403)$ \\
\hline 1353 & $\boldsymbol{B}$ & $\mathbf{m}$ & $v_{5}$ \\
\hline $\int 1079$ & $A$ & vs & $v_{6}$ \\
\hline$+\{1074$ & $C$ & w-m & $v_{13}$ \\
\hline 941 & $A$ & $\mathbf{s}$ & \\
\hline $914^{b}$ & $A$ & $\mathbf{s}$ & $2 v_{14}(924)$ \\
\hline 581 & $*$ & vw & $2 v_{9}(582)$ \\
\hline 565 & $A / B$ & $\mathbf{s}$ & $v_{9}+v_{10}(562)$ \\
\hline 545 & $B$ & vs & $v_{8}$ \\
\hline $462^{b}$ & $C$ & vw & $v_{14}$ \\
\hline 291 & $A$ & $\mathbf{w}$ & $v_{9}$ \\
\hline 271 & $\boldsymbol{A}$ & $\mathbf{w}$ & $v_{10}$ \\
\hline
\end{tabular}

a The band from the $C_{s}$-rotamer is supposed to be hidden in the band from the $C_{1}$-rotamer. ${ }^{b}$ The most intense of several $Q$-branches.
Table 2. Infrared gas phase data and vibrational assignment for acetyliodide- $d_{3}$. For symbols used see legend to Table 1. For footnotes see Table 1.

\begin{tabular}{clll}
$\begin{array}{c}\text { Frecuency } \\
\left(\mathrm{cm}^{-1}\right)\end{array}$ & $\begin{array}{l}\text { Band } \\
\text { contour }\end{array}$ & $\begin{array}{l}\text { Inten- } \\
\text { sity }\end{array}$ & $\begin{array}{l}\text { Assign- } \\
\text { ment }\end{array}$ \\
\hline 3604 & $*$ & w & $2 v_{3}(3634)$ \\
2746 & $A / B$ & vw & $v_{3}+v_{7}(2755)$ \\
2616 & $A / B$ & vw & $v_{3}+v_{6}(2616)$ \\
2265 & $A$ & vw & $v_{1}$ \\
2104 & $A / B$ & vw & $v_{3}+v_{9}(2105)$ \\
2034 & $A / B$ & vw & $v_{5}+v_{7}(2046)$ \\
1903 & $B$ & vw & $v_{5}+v_{6}(1907)$ \\
1873 & $A / B$ & m & $2 v_{7}(1876)$ \\
1817 & $B$ & vs & $v_{3}$ \\
1300 & $C$ & vw & $v_{9}+v_{12}(1325)$ \\
$1108^{b}$ & $A$ & vs & $v_{5}$ \\
$\int_{1052}$ & $A / B$ & w & $v_{4}$ \\
1037 & $C$ & w & $v_{12}$ \\
1020 & $A / B$ & w & $v_{6}+v_{10}(1039)$ \\
935 & $A / B$ & vs & $v_{7}$ \\
799 & $A$ & w & $v_{6}$ \\
529 & $*$ & w & $v_{9}+v_{10}(528)$ \\
500 & $A / B$ & vs & $v_{8}$ \\
$414^{b}$ & $C$ & vw & $v_{14}$ \\
288 & $A$ & w & $v_{9}$ \\
240 & $A / B$ & w & $v_{10}$ \\
\hline & & &
\end{tabular}

Fig. 3 shows the $\mathrm{CH}$-stretching region for the AcI, AcI- $d_{1}$ and AcI- $d_{2}$. All the spectra are of saturated vapours recorded at room temperature in a $10 \mathrm{~cm}$ cell, and are presented in transmission. For the parent molecule three bands (two $A / B$ hybrids and one $C$-type band) would be expected. ${ }^{12,13}$ Only two bands, of pronounced $A$-type, are observed at $3014 \mathrm{~cm}^{-1}$ and at 2937 $\mathrm{cm}^{-1}$, and they are assigned to the asymmetric and symmetric $\mathrm{CH}$-stretching modes, respectively $\left(v_{1}\right.$ and $\left.v_{2}, A^{\prime}\right) .{ }^{13}$ The missing $C$-type band $\left(v_{11}\right.$, asymmetric stretch, $\left.A^{\prime \prime}\right)$ might be weak and/or coincident with the Q-branch in $v_{1}$. In the $C_{3 v}$-case these two bands $\left(v_{1}\right.$ and $\left.v_{11}\right)$ are degenerate.

In the $d_{1}$-species a total of four bands are expected in the $\mathrm{CH}$-stretching region; an $A / B$ and a $C$-type band from the $C_{s}$ rotamer and two $A / B / C$-hybrid bands from the $C_{1}$ rotamer. Only two pronounced $A$-type bands of nearly equal intensity are observed, as shown in Fig. 3c.

For the $d_{2}$-species one $A / B$-band and one $A / B / C$-band from the $C_{s}$ and the $C_{1}$ rotamer respectively, would be expected. But in fact only 
Table 3. Infrared gas phase data and vibrational assignment for acetyliodide- $d_{1}$. For symbols used see legend to Table 1. For footnotes see Table 1.

\begin{tabular}{|c|c|c|c|c|c|}
\hline $\begin{array}{l}\text { Frequency } \\
\left(\mathrm{cm}^{-1}\right)\end{array}$ & $\begin{array}{l}\text { Band } \\
\text { contour }\end{array}$ & $\begin{array}{l}\text { Inten- } \\
\text { sity }\end{array}$ & \multicolumn{3}{|c|}{$\underset{C_{1}}{\text { Assignment }}$} \\
\hline 3600 & $B$ & vw & $2 v_{3}(3626)$ & $2 v_{2}(3626)$ & \\
\hline 3007 & $A$ & vw & & $v_{1}$ & \\
\hline$+\{2958$ & $A$ & vw & & $v_{2}$ & \\
\hline$+\{2953$ & $*$ & vvw & & & \\
\hline $\int 2769$ & $B$ & vvw & $v_{3}+v_{7}(2775)$ & $v_{4}+v_{9}(2775)$ & \\
\hline$+\{2754$ & $B$ & vvw & & $v_{3}+v_{11}(2768)$ & \\
\hline 2640 & $B$ & vw & $v_{4}+v_{5}(2658)$ & $v_{4}+v_{10}(2640)$ & \\
\hline ca. 2223 & $*$ & vvw & $\begin{array}{l}v_{2} \\
v_{7}+v_{12}(2228)\end{array}$ & & \\
\hline 2100 & $*$ & vw & $v_{3}+v_{9}(2103)$ & $\begin{array}{l}2 v_{8}(2104) \\
v_{4}+v_{13}(2103) \\
v_{6}+v_{10}(2101)\end{array}$ & \\
\hline 1922 & $A / B$ & $\mathbf{w}$ & $2 v_{7}(1924)$ & $2 v_{9}(1924)$ & \\
\hline$+\left\{1891^{\text {sh }}\right.$ & $A$ & $\mathbf{m}$ & $v_{4}+v_{8}(1913)$ & & \\
\hline$+\{1880$ & $B$ & $\mathrm{~m}$ & & $v_{8}+v_{10}(1879)$ & \\
\hline 1858 & $A$ & $\mathrm{~m}$ & $v_{6}+v_{7}(1873)$ & & F \\
\hline 1813 & $B$ & vs & & $v_{4}$ & \\
\hline ca. 1655 & * & vvw & $v_{4}+v_{10}(1670)$ & $2 v_{10}(1654)$ & \\
\hline ca. 1590 & $*$ & vVw & & $v_{8}+v_{11}(1597)$ & \\
\hline $\int 1429$ & $B$ & $\mathbf{w}$ & $v_{6}+v_{8}(1415)$ & & \\
\hline$+\{1398$ & $A$ & $\mathbf{w}$ & $v_{4}$ & & \\
\hline |1412 & $C$ & $\mathbf{w}$ & & $v_{5}$ & \\
\hline ca. 1300 & $*$ & vw & $v_{10}+v_{13}(1305)$ & $v_{8}+v_{14}(1312)$ & \\
\hline $\int 1274$ & $A$ & m & & $v_{6}$ & \\
\hline 1266 & $C$ & $\mathrm{~m}$ & $v_{12}$ & & \\
\hline${ }^{+} \mid 1260$ & $A$ & m & $v_{5}$ & & \\
\hline 1249 & $\boldsymbol{B}$ & m & & $v_{7}$ & \\
\hline 1079 & $C$ & $\mathbf{w}$ & & $2 v_{11}(1090)$ & \\
\hline 1071 & $C$ & $\mathbf{w}$ & & $v_{10}+v_{14}(1086)$ & \\
\hline$+\{1052$ & $A / B / C$ & vs & & $v_{8}$ & \\
\hline+ T1045 & $C$ & vs & $v_{13}$ & & \\
\hline 962 & $A / B$ & vs & $v_{7}$ & & \\
\hline 911 & $A$ & $\mathbf{w}$ & $v_{6}$ & & \\
\hline 827 & $A$ & $\mathbf{w}$ & & $v_{10}$ & \\
\hline 552 & $A / B$ & $\mathbf{s}$ & & $v_{3}+v_{14}(549)$ & $\mathrm{F}$ \\
\hline 540 & $A$ & vs & & $v_{11}$ & $F$ \\
\hline $504^{b}$ & $A$ & $\mathbf{s}$ & $v_{8}$ & & \\
\hline 290 & $A$ & $\mathbf{w}$ & $\nu_{9}^{\mathrm{a}}$ & $v_{13}$ & \\
\hline 261 & $A$ & $\mathbf{w}$ & $v_{10}$ & & \\
\hline 259 & $A$ & $\mathbf{w}$ & & $v_{14}$ & \\
\hline
\end{tabular}

a single pronounced $A$-type band is observed.

The absence of bands might be explained by assuming exact or nearly exact coincidence between bands from the two rotamers. This means that the methyl group has $C_{3 v}$ or very near $C_{3 v}$ symmetry. However, a very small deviation from $C_{3 v}$ symmetry should cause a frequency difference of a few wave numbers, which should be observable. Another explanation of the missing bands, including the absence of the $A^{\prime \prime} \mathrm{CH}-$ stretching mode in AcI may be as follows.

First the overall weak intensity of the $\mathrm{CH}$ stretching bands must be caused by a small value of the dipole moment derivative $(\delta \vec{\mu} / \delta r)_{0}$, where $r$ is the $\mathrm{CH}$ distance. Secondly, excluding that the low intensity of the $A^{\prime \prime} \mathrm{CH}$-stretching mode is 
Table 4. Infrared gas phase data and vibrational assignment for acetyliodide- $d_{2}$. For symbols used see legend to Table 1. For footnotes see Table 1.

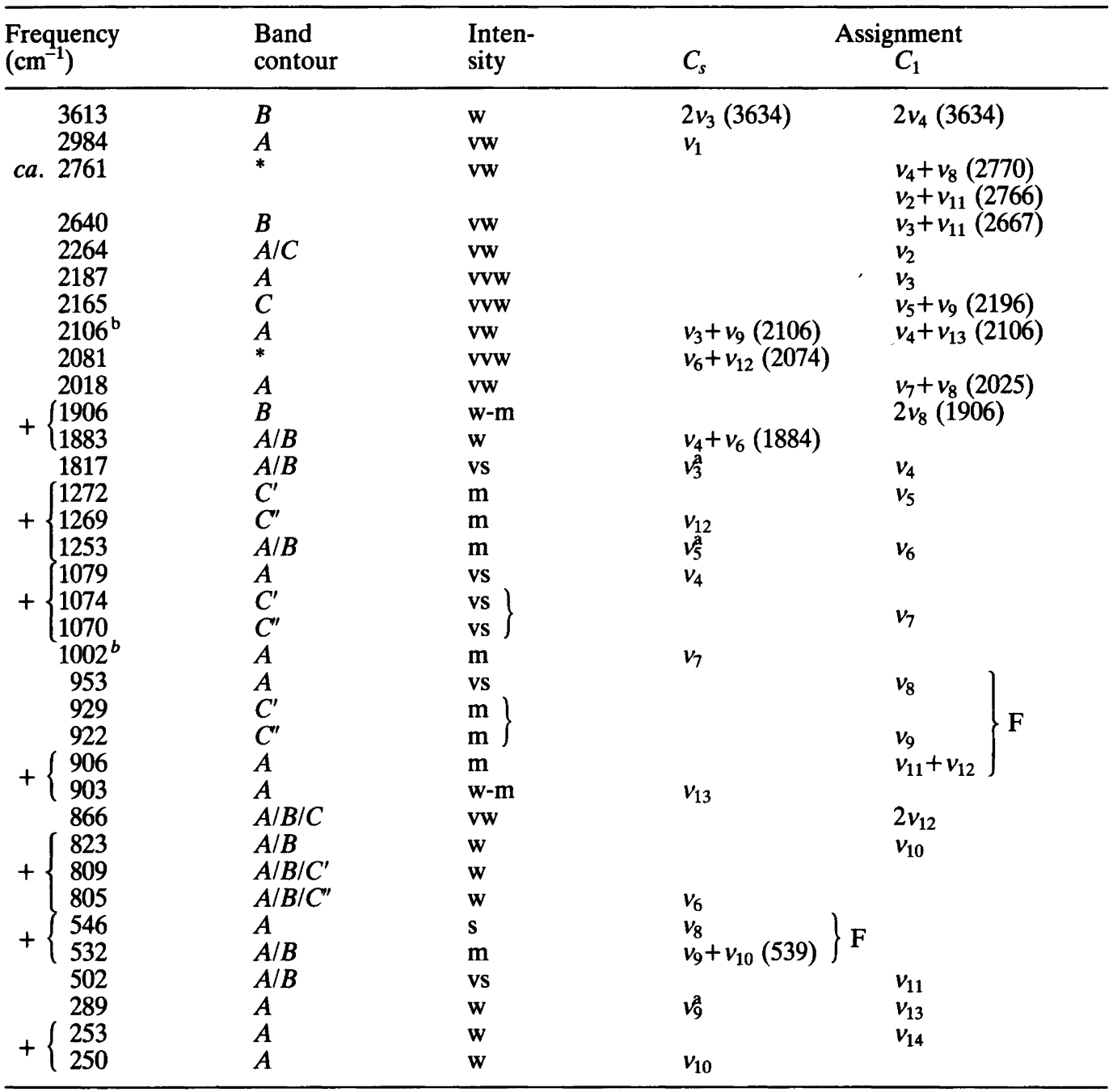

accidental, $(\delta \vec{\mu} / \delta r)_{0}$ must be significantly larger for the $\mathrm{CH}$-bond in the plane defined by the heavy atoms than for the two other (equivalent) $\mathrm{CH}$-bonds. As a consequence, only vibrations involving the "in plane" $\mathrm{CH}$-bond would be expected to give rise to observable bands, which is in accordance with the number of observed bands. Moreover, because the dipole moment change will be mainly along the "in plane" CH-bond, which is parallel with the $a$-axis (Fig. 1) all bands will appear as $A$-type as is in fact observed. Finally the theory qualitatively pre- dicts the relative intensities of the observed bands. The assignment according to this explanation is given in Tables 1-4.

CH/CD-bendings and skeletal vibrations. AcI and $A C I-d_{3}$. The remaining assignment of the parent species requires only a few comments. The assignment is given in Table 1 . However, several Fermi resonances occur, and corrections are necessary in order to get the "correct" fundamental frequencies. In Fig. 4a, c and d these Fermi resonances are shown. The corrections are carried out after the method described Acta Chem. Scand. A 38 (1984) No. 6 

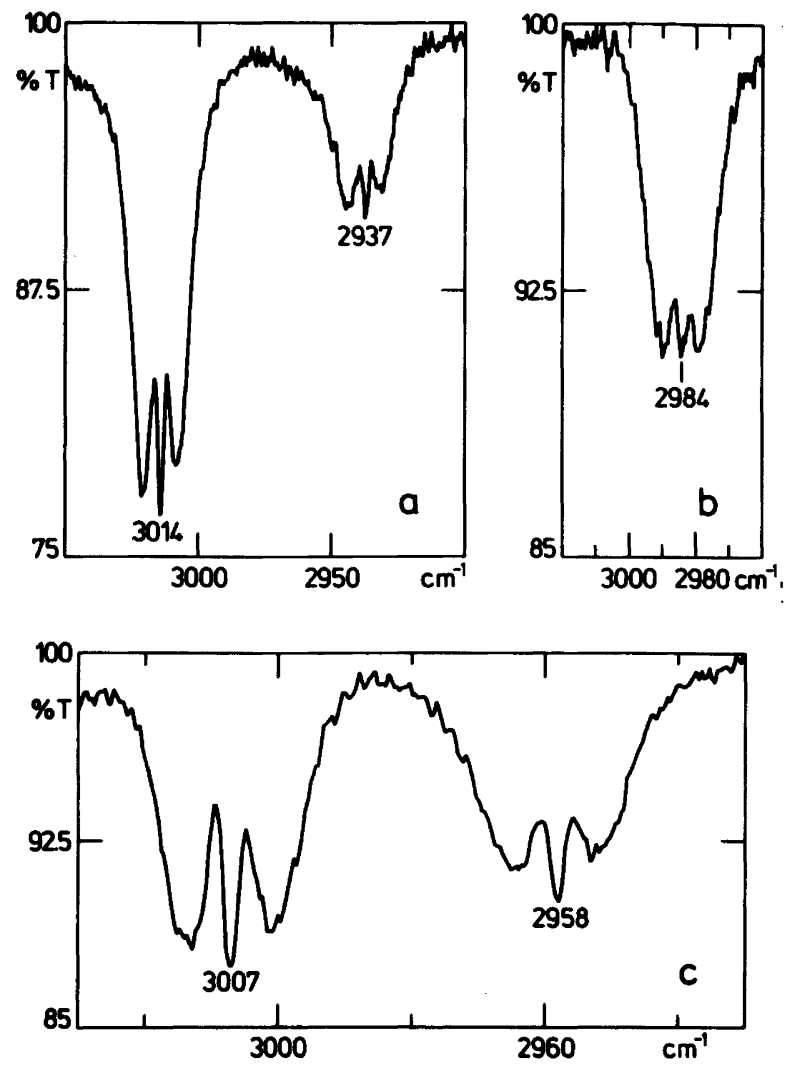

Fig. 3. Infrared gas spectra of the $\mathrm{CH}$ stretching region for a. AcI, b. AcI- $d_{2}$ and c. AcI- $d_{1}$ (saturated vapours in a $10 \mathrm{~cm}$ cell).

in Ref. 14 and by using intensity data from spectra recorded in absorbance. The corrected values are given in Table 7. The Fermi resonance near $950 \mathrm{~cm}^{-1}$ shown in Fig. $4 \mathrm{c}$ is assigned to two fundamentals of $A^{\prime}$ - and $A^{\prime \prime}$-symmetry, respectively in Ref. 2, probably because of spectra of poorer quality. Fig. $4 \mathrm{c}$ shows clearly that two $A$-type bands are present, and only a Fermi resonance can explain this. The $A^{\prime \prime}$-fundamental ( $v_{13}, \mathrm{CH}_{3}$-rock), assigned to one of the Fermi doublets in Ref. 2, appears as a weak $C$-type band at $1074 \mathrm{~cm}^{-1}$ overlapping the $A^{\prime}$-fundamental ( $v_{6}, \mathrm{CH}_{3}$-rock) at $1079 \mathrm{~cm}^{-1}$ (Fig. 4b). The two modes $\left(v_{6}\right.$ and $\left.v_{13}\right)$ are degenerate in the $C_{3 v}$-case and are supposed to be close in frequency. In the far-IR two slightly separated $A$-type bands are observed, Fig. 5a. They cannot be explained as a Fermi resonance, and must therefore be attributed to two skeletal bending modes.
This assignment is confirmed from the deuterated species. The high frequency band, $v_{9}$, is nearly insensitive to deuteration in contrast to the low frequency band, $v_{10}$, (Fig. $5 a$ and b). This indicates that the $\mathrm{CH}_{3}$-group participates in the $v_{10}$ mode and not in the $v_{9}$ mode.

For the $d_{3}$-species the remaining assignment is also rather straightforward. The assignment is given in Table 2 . It is worth mentioning that the $\mathrm{CD}_{3}$-rock vibration of $A^{\prime \prime}$-symmetry $\left(v_{13}\right)$ is totally absent. The $C$-type band at $414 \mathrm{~cm}^{-1}$ is assigned to the out of plane skeletal deformation, $v_{14}$. This band is very weak. In the parent species the corresponding band is observed at $462 \mathrm{~cm}^{-1}$, but it is not as sharp and distinct as in AcI- $d_{3}$. The corresponding bands are not observed in the spectra of AcI- $d_{1}$ and AcI- $d_{2}$ due to insufficient intensity.

$A c I-d_{1}$ and $A c I-d_{2}$. In contrast to the parent- 

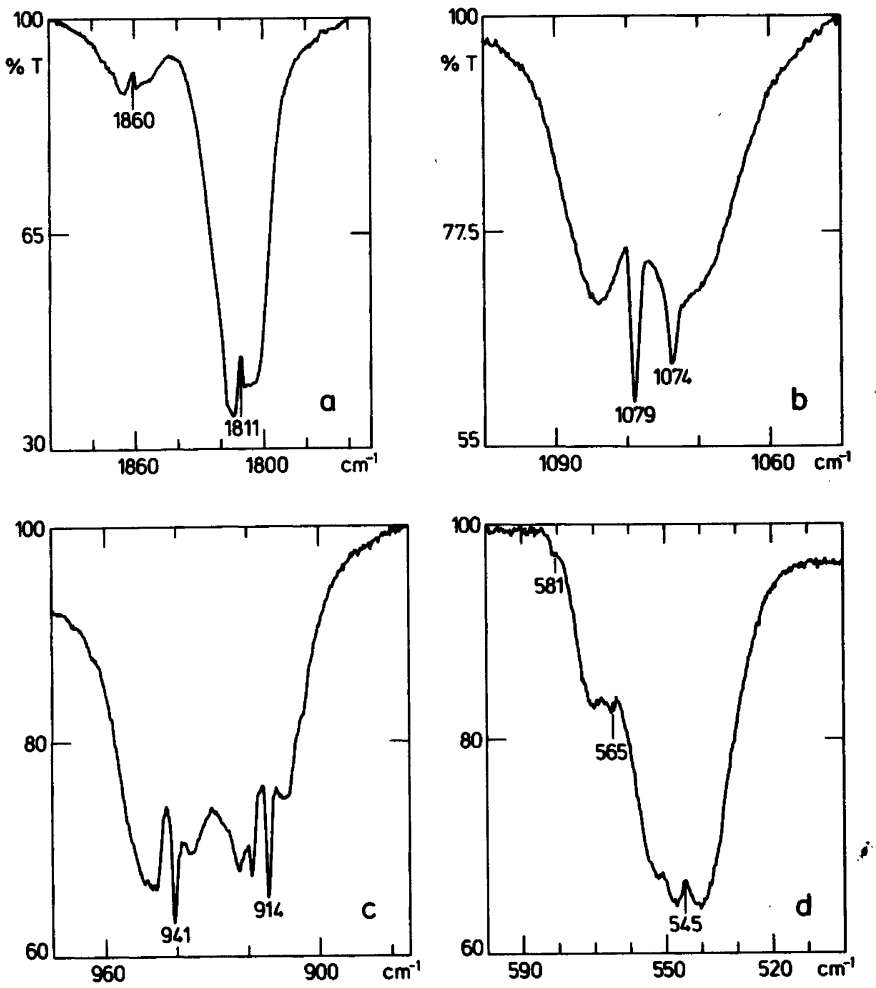

Fig. 4. Details of the infrared gas spectra of acetyliodide. a., c. and d., important Fermi resonance. b. The near degenerate modes $v_{6}$ and $v_{13}$.

and the $d_{3}$-species the assignment of the $d_{1}$ - and the $d_{2}$-species is quite difficult. The presence of two rotamers doubles the number of bands and gives rise to many overlapping bands. Furthermore, due to the low symmetry of the $C_{1}$ rotamer the band contours are of less help in the assignment. A reasonable assignment based on the present experimental material can, in our opinion only be carried out after good estimates of fundamental bands for the involved species have been made. The assignment of the $d_{0^{-}}$and the $d_{3}$-species and of the CH-stretching frequencies for the $d_{1}$ - and the $d_{2}$-species is used to establish a simple valence force field for acetyliodide (see below), and from this the fundamental frequencies for the $d_{1}$ - and $d_{2}$-species are calculated. The assignment obtained in this way is given in Tables 3 and 4. A detailed discussion will not be given here, but in Figs. 6 and 7 details are shown of the more complicated regions, where the assignment is less certain.
In cases, where bands from the $C_{1}$ and the $C_{s}$ rotamer are calculated as being only slightly separated, and only one band is observed, this band is assigned to the $C_{1}$ rotamer. The band from the $C_{s}$ rotamer, which is believed to have approximately half the intensity of the $C_{1}$ rotam$\mathrm{er}$, is in such cases assumed to be hidden in the $C_{1}$ band, and the frequency for the $C_{s}$ band is estimated to be near the $C_{1}$ value.

Force field calculations. Coordinates. The force field calculations are carried out after the GFmethod. ${ }^{8}$ Conventional internal valence coordinates are used with the exception that the $\mathrm{H}_{\mathbf{i}} \mathrm{CH}_{\mathbf{j}}$ angles are replaced by the corresponding dihedral angles, $d_{\mathrm{ij}}$, between the planes defined by the two carbon atoms and $\mathrm{H}_{\mathrm{i}}$ and $\mathrm{H}_{\mathrm{j}}$, respectively. The coordinates are named $\tau_{\mathrm{i}}$ after:

$\tau_{1}=d_{23} \quad \tau_{2}=d_{13} \quad \tau_{3}=d_{12}$

The advantage of these coordinates is discussed in Ref. 15. The torsion of the methyl group is 

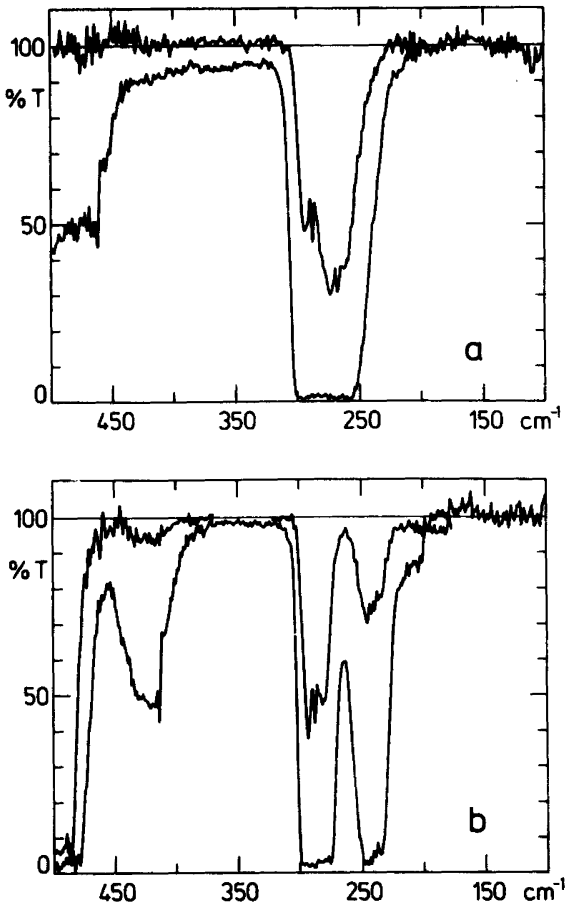

Fig. 5. The far infrared gas spectra of acetyliodide and acetyliodide- $d_{3}$ at two different pressures (ca. 2 and ca. $13 \mathrm{mmHg}$ in a 4.6 meter cell). described by the torsions of the individual $\mathrm{H}_{\mathrm{i}} \mathrm{CCO}$-systems:

$t_{i}=\operatorname{tors}\left(\mathrm{H}_{i} \mathrm{CCO}\right) \quad i=1,2,3$

The out of plane skeletal deformation, $\gamma$, is defined as the angle between the CO-bond and the plane defined by CCI. The definition of the remaining coordinates is given in Fig. 1 and Table 9. The symmetry coordinates used $\left(C_{s}\right)$ are given in Table 5.

Calculations. A least squares method was applied in adjusting the force constants using a program developed at Chemical Laboratory $\mathrm{V}$, University of Copenhagen. The program is based on well known principles already described in several papers, ${ }^{16,17,18}$ and in particular we would draw attention to the papers by Aldous and Mills, ${ }^{16,17}$ since they include a fairly comprehensive discussion on the problem of error analysis.

The force constants are adjusted to fit the observed frequencies. The estimated probable error, $\sigma_{i}$, in the i'th observed frequency, $\omega_{i}$, is taken to be proportional to $\omega_{\mathrm{i}}$ and is set to $\sigma_{\mathrm{i}}=0.01 \omega_{\mathrm{i}}$.

A general force field has 55 independent force constants in the $A^{\prime}$-class and 15 in the $A^{\prime \prime}$-class,
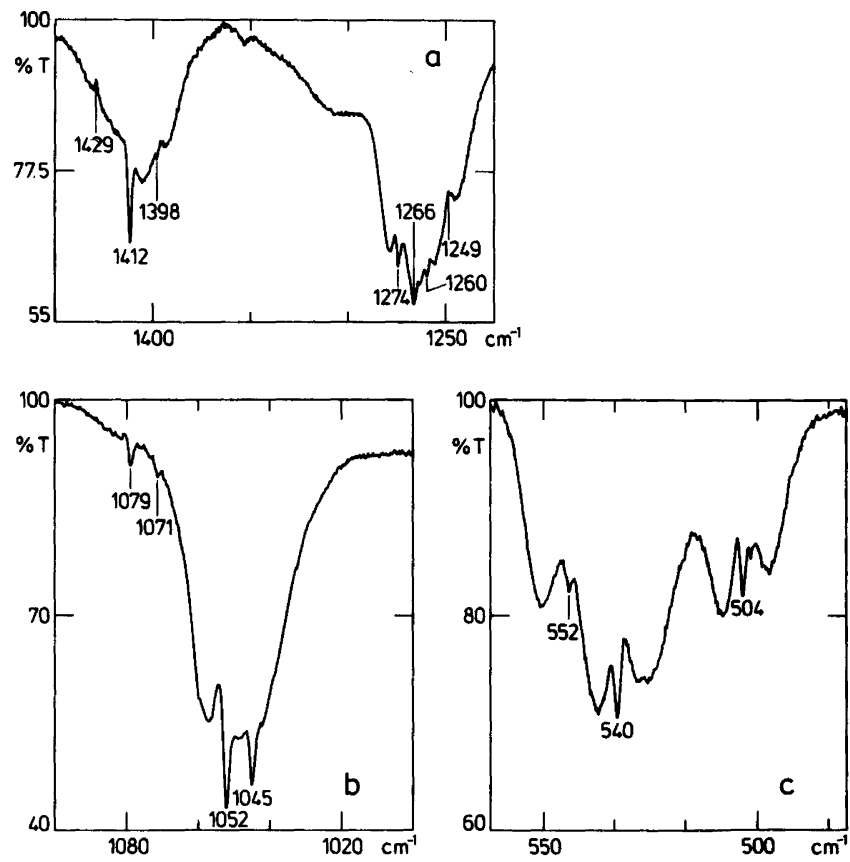

Fig. 6. Details of the infrared gas spectra of acetyliodide- $d_{1}$ where the assignment is uncertain. 

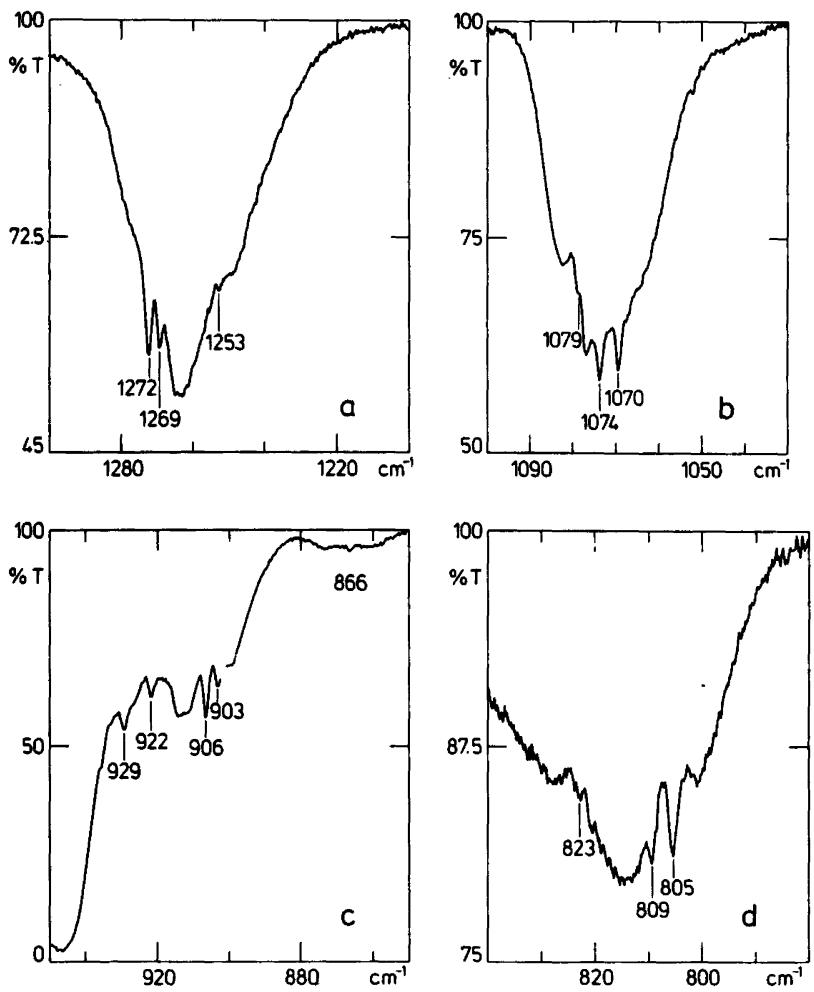

Fig. 7. Details of the infrared gas spectra of acetyliodide- $d_{2}$ where the assignment is uncertain.

and it is necessary to fix many of these to zero or literature values. The initial force field values are taken from the standard methyl group of Duncan, ${ }^{12} \mathrm{HCOCl},{ }^{19,20}$ and $\mathrm{CH}_{3} \mathrm{CHO},{ }^{21}$ and are

Table 5. Symmetry coordinates for acetyl iodide.

$$
\begin{array}{ll}
\hline A^{\prime} \quad S_{1}=6^{-1 / 2}\left(2 \Delta r_{1}-\Delta r_{2}-\Delta r_{3}\right) \\
S_{2}=6^{-1 / 2}\left(2 \Delta \tau_{1}-\Delta \tau_{2}-\Delta \tau_{3}\right) \\
S_{3}=6^{-1 / 2}\left(2 \Delta \beta_{1}-\Delta \beta_{2}-\Delta \beta_{3}\right) \\
S_{4}=3^{-1 / 2}\left(\Delta r_{1}+\Delta r_{2}+\Delta r_{3}\right) \\
S_{5}=3^{-1 / 2}\left(\Delta \beta_{1}+\Delta \beta_{2}+\Delta \beta_{3}\right) \\
S_{6}=\Delta R \\
S_{7}=\Delta u \\
S_{8}=\Delta w \\
S_{9}=2^{-1 / 2}(\Delta \varepsilon-\Delta \delta) \\
S_{10}=6^{-1 / 2}(2 \Delta \theta-\Delta \varepsilon-\Delta \delta) \\
A^{\prime \prime} \quad S_{11}=2^{-1 / 2}\left(\Delta r_{2}-\Delta r_{3}\right) \\
S_{12}=2^{-1 / 2}\left(\Delta \tau_{2}-\Delta \tau_{3}\right) \\
S_{13}=2^{-1 / 2}\left(\Delta \beta_{2}-\Delta \beta_{3}\right) \\
S_{14}=\Delta \gamma \\
S_{15}=3^{-1}\left(\Delta t_{1}+\Delta t_{2}+\Delta t_{3}\right)
\end{array}
$$

given in Table 6. The methyl group values given by Duncan ${ }^{12}$ are converted to the coordinates used here $\left(\tau^{\prime} \mathbf{s}\right)$. The constants not shown in Table 6 are set to zero.

On the asymmetry of the methyl group. The symmetry coordinates used for the methyl group (Table 5) transform according to $C_{3 v}$ symmetry, and $\left(S_{1}, S_{11}\right),\left(S_{2}, S_{12}\right)$ and $\left(S_{3}, S_{13}\right)$ transform as degenerate sets of E-species. However, it is well documented that the methyl group in acetyl halides deviate from local $C_{3 v}$-symmetry. ${ }^{3}$ McKean has derived the force constants for the individual $\mathrm{CH}$ stretching modes (trans and gauche to the halogen) from observed isolated $\mathrm{CH}$-stretching frequencies in $d_{2}$-acetyl halides. The asymmetry is shown to decrease, from being significant in AcF to nearly non-existent in AcI. From the extended material presented in this work, we wished to confirm the methyl group geometry.

The asymmetry of the methyl group force field is most clearly described when using internal 
Table 6. Initial and final force constants for AcI. I and II represent a severely restricted force field and a minimum restricted force field, respectively. For details see text.

\begin{tabular}{|c|c|c|c|}
\hline $\begin{array}{l}\text { Force } \\
\text { constant } t^{a, b}\end{array}$ & ' Initial & I & II \\
\hline$F_{1,1}$ & $4.82^{c}$ & $4.862(36)$ & $4.857(21)$ \\
\hline$F_{1,2}=F_{11,12}$ & $-0.10^{c}$ & -0.10 & -0.10 \\
\hline$F_{1,3}=F_{11,13}$ & $0.05^{c}$ & 0.05 & 0.05 \\
\hline$F_{1,4}$ & 0 & 0 & $-0.006(7)$ \\
\hline$F_{2,2}$ & $0.37^{c}$ & $0.354(3)$ & $0.352(4)$ \\
\hline$F_{2,3}$ & $0.23^{c}$ & $0.221(5)$ & $0.218(7)$ \\
\hline$F_{3,3}$ & $0.81^{c}$ & $0.775(16)$ & $0.769(35)$ \\
\hline$F_{3,5}$ & 0 & 0 & $-0.010(11)$ \\
\hline$F_{3,9}$ & 0 & $-0.105(14)$ & $-0.083(21)$ \\
\hline$F_{3,10}$ & 0 & $0.105(14)$ & $0.128(53)$ \\
\hline$F_{4,4}$ & $4.95^{c}$ & $4.988(44)$ & 4.984(19) \\
\hline$F_{4,5}$ & $0.04^{d}$ & 0.04 & 0.04 \\
\hline$F_{4,6}$ & $0.13^{d}$ & 0.13 & 0.13 \\
\hline$F_{5,5}$ & $1.18^{c}$ & $1.111(14)$ & $1.109(33)$ \\
\hline$F_{5,6}$ & $0.52^{c}$ & $0.427(25)$ & $0.411(30)$ \\
\hline$F_{6,6}$ & $4.45^{c}$ & $4.894(109)$ & $4.821(151)$ \\
\hline$F_{6,7}$ & $0.45^{e}$ & 0.450 & 0.45 \\
\hline$F_{6,8}$ & $0.68^{e}$ & 0.68 & 0.68 \\
\hline$F_{6,9}$ & $0.24^{e}$ & $0.546(79)$ & $0.506(87)$ \\
\hline$F_{6,10}$ & $0.10^{e}$ & $0.318(58)$ & $0.369(43)$ \\
\hline & $1.86^{g}$ & $1.591(78)$ & $1.677(24)$ \\
\hline$F_{7,8}$ & $0.55^{e}$ & 0.55 & 0.55 \\
\hline$F_{79}^{\prime \prime}$ & $-0.29^{e}$ & $-0.546(79)$ & -0.29 \\
\hline$F_{7,10}$ & $0.19^{e}$ & $0.318(58)$ & $0.369(43)$ \\
\hline & $13.20^{e}$ & $13.300(100)$ & $13.334(93)$ \\
\hline$F_{8,9}$ & $0.02^{e}$ & 0 & 0 \\
\hline$F_{8,10}$ & $-0.27^{e}$ & -0.27 & -0.27 \\
\hline$F_{9,9}$ & $0.79^{e}$ & $1.080(66)$ & $0.875(24)$ \\
\hline$F_{9,10}$ & $0.16^{e}$ & 0 & 0 \\
\hline$F_{10,10}$ & $0.68^{e}$ & $0.764(14)$ & $0.786(50)$ \\
\hline$F_{11,11}$ & $4.82^{c}$ & $4.862(36)$ & $4.868(12)$ \\
\hline$F_{12,12}$ & $0.37^{c}$ & $0.354(3)$ & $0.363(3)$ \\
\hline$F_{12,13}$ & $0.23^{c}$ & $0.221(5)$ & $0.209(5)$ \\
\hline$F_{13,13}$ & $0.81^{c}$ & $0.775(16)$ & 0.783(19) \\
\hline$F_{13,14}$ & 0 & $-0.146(14)$ & $-0.101(20)$ \\
\hline$F_{14,14}$ & $0.37^{f}$ & 0.474(19) & $0.442(20)$ \\
\hline$F_{15,15}$ & $0.037^{f}$ & 0.037 & 0.037 \\
\hline
\end{tabular}

a Units. stretch/stretch: $\mathrm{mdyn} / \AA=\mathrm{aJ} \AA^{-2}$; bend/bend: mdyn $\AA=a J$; stretch/bend: mdyn $=a J \AA^{-1}$. ${ }^{b}$ When no uncertainty is given the value has been fixed. ${ }^{c}$ Ref. 12 ${ }^{d}$ Ref. 22. ${ }^{e}$ Ref. 19. ${ }^{f}$ Ref. 21. ${ }^{g}$ Assumed.

coordinates. For AcI the first part of the general harmonic potential function involving the $\mathrm{CH}$ stretching coordinates is:

$$
2 V=f_{r}^{*} \Delta r_{1}^{2}+f_{r}\left(\Delta r_{2}^{2}+\Delta r_{3}^{2}\right)+
$$

$$
\begin{aligned}
& 2 f_{r r} \Delta r_{2} \Delta r_{3}+2 f_{r r}^{*} \Delta r_{1}\left(\Delta r_{2}+\Delta r_{3}\right)+ \\
& 2 f_{r \beta}^{*} \Delta r_{1} \Delta \beta_{1}+2 f_{r \beta}\left(\Delta r_{2} \Delta \beta_{2}+\Delta r_{3} \Delta \beta_{3}\right)+ \\
& 2 f_{r \beta}^{\prime *} \Delta r_{1}\left(\Delta \beta_{2}+\Delta \beta_{3}\right)+2 f_{r \beta}^{\prime}\left(\Delta r_{2} \Delta \beta_{3}+\Delta r_{3} \Delta \beta_{2}\right)+\ldots
\end{aligned}
$$

The force constants connected to the internal coordinates involving the "in plane" hydrogen atom are marked with an asterisk.

In the case of local $C_{3 v}$-symmetry $f_{r}^{*}=f_{\mathrm{r}}, f_{\mathrm{rr}}^{*}=f_{\mathrm{rr}}$ etc. It is seen that in the general case, the part of the potential function shown above contains eight different force constants instead of four different force constants in the $C_{3 v}$-case. The potential function must therefore be simplified before it can be used in force field refinement.

A useful approximation is obtained if one assumes that the asymmetry appears only in the quadratic terms $\left(\Delta r_{1}^{2}\right.$ etc.) and not in the mixed terms $\left(\Delta r_{1} \Delta r_{2}\right.$ etc.). This means that $f_{r}^{*} \neq f_{r}$, but $f_{r r}^{*}=f_{r r}, f_{r \beta}=f_{r \beta}^{*}$ and $f_{r \beta}^{*}=f_{r \beta}^{\prime}$. For the total potential function it means that the deviation from local $C_{3 v}$-symmetry is described by adding only three extra force constants $\left(f_{r}^{*}, f_{\beta}^{*}\right.$ and $\left.f_{\tau}^{*}\right)$.

The influence of this approximation on the symmetry force constants is given below for the coordinates involving the $\mathrm{CH}$-stretchings:

$$
\begin{aligned}
& F_{1,1}\left(F_{2,2}, F_{3,3}\right)=f_{r}-f_{r r}+2 \Delta f_{\mathrm{r}} / 3 \\
& F_{4,4}\left(F_{5,5}\right)=f_{r}+2 f_{r r}+\Delta f_{\mathrm{r}} / 3 \\
& F_{11,11}\left(F_{12,12}, F_{13,13}\right)=f_{r}-f_{r r} \\
& F_{1,4}\left(F_{3,5}\right)=2^{\frac{1}{2}} \Delta f_{r} / 3 \\
& \Delta f_{r}=f_{r}^{*}-f_{r}
\end{aligned}
$$

Corresponding formulae are obtained for the symmetry coordinates involving the $\tau$ 's and the $\beta$ s. The corresponding symmetry force constants are given in parenthesis in the formulae above.

It appears that in this approximation the symmetry force constants are correlated:

$$
\begin{aligned}
& F_{1,1}-F_{11,11}=2 \Delta f_{\mathrm{r}} / 3=2^{\frac{1}{2}} F_{1,4} \\
& F_{3,3}-F_{13,13}=2 \Delta f_{\beta} / 3=2^{\frac{1}{2}} F_{3,5}
\end{aligned}
$$


Table 7. Fundamental frequencies for AcI, AcI- $d_{3}$, AcI- $d_{1}$ and AcI- $d_{2}$, and the deviation between observed and calculated values for various refinements (I-IV, see text). If Obs.-Calc. is in parenthesis the frequency has not been used in the refinement.

\begin{tabular}{|c|c|c|c|c|c|c|c|c|c|c|}
\hline & Obs. & & Obs & Calc. & & Obs. & & Obs & -Calc. & \\
\hline & $\mathrm{cm}^{-1}$ & I & II & III & IV & $\mathrm{cm}^{-1}$ & I & II & III & IV \\
\hline & Acety & & & & & Acety & & & & \\
\hline$v_{1}$ & 3014 & 3 & 5 & 6 & 3 & $2265^{\circ}$ & (27) & (28) & (30) & (25) \\
\hline$v_{2}$ & 2937 & 1 & 2 & 1 & 0 & $2109^{c}$ & & & & \\
\hline$v_{3}$ & $1817^{a}$ & -2 & -2 & -2 & -2 & 1817 & 2 & 2 & 2 & 1 \\
\hline$v_{4}$ & 1421 & -7 & -1 & -3 & -5 & $1037^{a}$ & (7) & (12) & (11) & (10) \\
\hline$v_{5}$ & 1353 & -2 & -2 & -4 & -4 & 1108 & 1 & -2 & -2 & -1 \\
\hline$v_{6}$ & 1079 & -3 & -1 & -2 & -4 & 799 & 1 & 2 & 2 & 1 \\
\hline$v_{7}$ & $928^{a}$ & -1 & -2 & -3 & -4 & 935 & -2 & 1 & -1 & 0 \\
\hline$v_{8}$ & $551^{a}$ & 3 & 3 & 3 & 3 & 500 & -1 & -1 & -1 & -1 \\
\hline$v_{9}$ & 291 & 0 & 0 & 0 & 0 & 288 & 0 & 0 & 0 & 0 \\
\hline$v_{10}$ & 271 & 1 & 0 & 1 & 1 & 240 & 0 & -1 & -1 & -1 \\
\hline$v_{11}$ & $3011^{c}$ & & & & & $2236^{c}$ & & & & \\
\hline$v_{12}$ & 1429 & -3 & -7 & -8 & -8 & 1037 & 7 & 2 & 1 & -1 \\
\hline$v_{13}$ & 1074 & 2 & 0 & -2 & -1 & $882^{b}$ & (14) & (2) & $(-3)$ & $(-7)$ \\
\hline$v_{14}$ & 462 & & & & 1 & 414 & 1 & & -1 & -1 \\
\hline$v_{15}$ & $134^{c}$ & & & & & $96^{c}$ & & & & \\
\hline & Acety & $e-d_{1}, C$ & symm & & & Acety & $d_{1}$ & symm & & \\
\hline & $2964^{c}$ & & & & & 3007 & & -3 & -4 & -2 \\
\hline$v_{2}$ & $2191^{c}$ & & & & & 2958 & & -4 & -1 & 0 \\
\hline$v_{3}$ & $1818^{c}$ & & & & & $2192^{c}$ & & & & \\
\hline$v_{4}$ & $1409^{a}$ & (1) & 4 & 3 & 3 & $1818^{c}$ & & & & \\
\hline$v_{5}$ & 1260 & $(-4)$ & -8 & -7 & -8 & 1412 & & -1 & 6 & 10 \\
\hline$v_{6}$ & 911 & $(-1)$ & 7 & 5 & 5 & 1274 & & 7 & 7 & 8 \\
\hline$v_{7}$ & 962 & (13) & 10 & 8 & 8 & 1249 & & -3 & -1 & -1 \\
\hline$v_{8}$ & 504 & -1 & 1 & 0 & 0 & 1052 & & 0 & 5 & 6 \\
\hline$v_{9}$ & 290 & (2) & 0 & 0 & 0 & $987^{c}$ & & & & \\
\hline$v_{10}$ & 261 & (0) & -1 & 0 & 0 & 827 & & -6 & -5 & -5 \\
\hline$v_{11}$ & $3011^{c}$ & & & & & $545^{a}$ & & 0 & 0 & 0 \\
\hline$v_{12}$ & 1266 & $(-1)$ & 5 & 4 & 4 & $436^{c}$ & & & & \\
\hline$v_{13}$ & 1045 & (19) & 10 & 8 & 7 & 290 & & 0 & 0 & 0 \\
\hline$v_{14}$ & $458^{c}$ & & & & & 259 & & -1 & -2 & -2 \\
\hline$v_{15}$ & $116^{c}$ & & & & & $117^{c}$ & & & & \\
\hline & Acety & $e-d_{2}, C$ & symm & & & Acety & $-d_{2}$ & symm & & \\
\hline$v_{1}$ & 2984 & -4 & -1 & -1 & -1 & $2989^{c}$ & & & & \\
\hline$v_{2}$ & $2151^{c}$ & & & & & 2264 & & (27) & (27) & (28) \\
\hline$v_{3}$ & 1817 & (1) & 1 & 1 & 0 & 2187 & & (38) & (40) & (41) \\
\hline$v_{4}$ & 1079 & $(-4)$ & -6 & -6 & -7 & 1817 & & 0 & 0 & 1 \\
\hline$v_{5}$ & $1249^{c}$ & & & & & 1272 & & 8 & 6 & 6 \\
\hline$v_{6}$ & 805 & $(-4)$ & 3 & 3 & 3 & 1253 & & (10) & (1) & (2) \\
\hline$v_{7}$ & 1002 & -1 & 1 & 0 & 2 & 1072 & & 9 & 9 & 10 \\
\hline$v_{8}$ & $540^{a}$ & $(0)$ & -2 & -1 & -1 & 953 & & -10 & -6 & -5 \\
\hline$v_{9}$ & 289 & $(-2)$ & 0 & 0 & 0 & 925 & & -7 & -6 & -6 \\
\hline$v_{10}$ & 250 & (3) & 2 & 2 & 3 & 823 & & -5 & -4 & -2 \\
\hline$v_{11}$ & $2236^{c}$ & & & & & 502 & & 0 & 0 & 0 \\
\hline$v_{12}$ & 1269 & (7) & -5 & -6 & -6 & $434^{c}$ & & & & \\
\hline$v_{13}$ & 903 & (2) & (21) & (16) & (13) & 289 & & 0 & 0 & 0 \\
\hline$v_{14}$ & $418^{c}$ & & & & & 253 & & 1 & 1 & 0 \\
\hline$v_{15}$ & $106^{c}$ & & & & & $104^{c}$ & & & & \\
\hline
\end{tabular}

${ }^{a}$ Corrected for Fermi resonance. ${ }^{b}$ Calculated from the product rule. ${ }^{c}$ Calculated in iteration sequence II. 
The calculations described below are all carried out in such a way that this correlation is fulfilled.

Iteration procedure. The fundamental frequencies calculated from the initial force field were in near agreement with the assignment of the parent and the $d_{3}$-species, and it was suitable as a starting point for further refinements.

Iteration sequence $I$. In this first iteration sequence severe constraints were used. The methyl group force field was constrained to $C_{3 v}$ symmetry. According to eqn. $1-7$, with $\Delta f=0$, this yields

$F_{1,1}=F_{11,11} \quad F_{2,2}=F_{12,12} \quad F_{3,3}=F_{13,13}$

$F_{2,3}=F_{12,13} \quad F_{1,4}=F_{3,5}=0$
The stretch/bend and bend/bend interaction constants of the skeleton were constrained to $C_{2 v}$ symmetry with the $\mathrm{CO}$ bond as the $C_{2}$-axis:

$F_{6,9}=-F_{7,9} \quad F_{6,10}=F_{7,10} \quad F_{9,10}=0$

Moreover some force constants were fixed on initial values, or if they turned out to be small and uncertain, to zero. In order to obtain convergence it proved to be necessary to introduce interaction terms between the methyl deformation mode $S_{3}$ and the skeleton bendings $S_{9}$ and $S_{10}$. Because they appeared with different sign they were constrained to $F_{3,9}=-F_{3,10}$.

Only frequencies where the assignment is unequivocal were used in this iteration; i.e. all assigned frequencies from the $d_{0^{-}}$and the $d_{3^{-}}$

Table 8. Calculated internal force constants for the methyl group deformations for various refinements. II, III, and IV only differ in the methyl group structure.

\begin{tabular}{lllll}
\hline $\begin{array}{llll}\text { Force } \\
\text { constants }\end{array}$ & \multicolumn{4}{c}{ Iteration sequence (see text) } \\
\cline { 2 - 5 } & $\mathrm{I}^{b}$ & $\mathrm{II}^{b}$ & $\mathrm{III}^{c}$ & IV $^{d}$ \\
\hline$f_{r}$ & $4.904(51)$ & $4.908(9)$ & $4.902(9)$ & $4.895(8)$ \\
$f_{r r}$ & $0.042(27)$ & $0.041(3)$ & $0.034(3)$ & $0.035(2)$ \\
$\Delta f_{r}$ & 0 & $-0.016(14)$ & $-0.006(14)$ & $-0.004(12)$ \\
$f_{\beta}$ & $0.887(21)$ & $0.894(13)$ & $0.892(12)$ & $0.890(10)$ \\
$f_{\beta \beta}$ & $0.112(10)$ & $0.111(6)$ & $0.112(6)$ & $0.114(5)$ \\
$\Delta f_{\beta}$ & 0 & $-0.021(24)$ & $-0.013(22)$ & $-0.001(19)$ \\
$\Delta f_{\tau}$ & 0 & $-0.011(7)$ & $-0.011(6)$ & $-0.004(6)$ \\
\hline
\end{tabular}

${ }^{a}$ For units see Table 6. ${ }^{b} \beta=108.1^{\circ}, \alpha=110.8 .{ }^{c} \beta=\alpha=109.47^{\circ} .{ }^{d} \beta=110.1^{\circ}, \alpha_{2}=\alpha_{3}=110.34^{\circ}$.

Table 9. Structure for AcI used in the force field refinements I-IV.

\begin{tabular}{lllll}
\hline $\begin{array}{l}\text { Bond or } \\
\text { angle }\end{array}$ & Symbol & $\begin{array}{l}\text { Value }^{a} \\
\mathrm{I,II}^{b}\end{array}$ & III & IV \\
\hline CH1 (in plane) & $\mathrm{r}_{1}$ & 1.101 & - & - \\
CH2/CH3 & $\mathrm{r}_{2} / \mathrm{r}_{3}$ & 1.101 & - & - \\
H2CH3 & $\alpha_{1}$ & 110.81 & 109.47 & 105.84 \\
H1CH2/H1CH3 & $\alpha_{3} / \alpha_{2}$ & & & 110.34 \\
H1CC & $\beta_{1}$ & 108.10 & 109.47 & 110.10 \\
H2CC/H3CC & $\beta_{2} / \beta_{3}$ & 1.492 & - & - \\
CC & $\mathrm{R}$ & 1.198 & - & - \\
CO & $\mathrm{w}$ & 2.217 & - & - \\
CI & $\mathrm{u}$ & 128.8 & - & - \\
CCO & $\varepsilon$ & 111.7 & - & - \\
CCI & $\theta$ & 119.5 & - & - \\
OCI & $\delta$ & & & \\
\hline
\end{tabular}

\footnotetext{
${ }^{a}$ Bond lengths in $\AA$ and angles in degrees. ${ }^{b}$ Ref. 5.
} 
species, $v_{8}$ from the $d_{1}$-species $\left(C_{s}\right)$, and $v_{1}$ and $v_{7}$ from the $d_{2}$-species $\left(C_{s}\right)$. The result is shown in Tables 6 and 7 , column I.

Iteration sequence II. From the force field obtained above the frequencies were calculated for the $d_{1}$ and the $d_{2}$ species ( $C_{1}$ and $C_{s}$ rotamers) and the complete assignment given in the Tables 3 and 4 was carried out.

The new assigned frequencies were then introduced in a new iteration sequence. Some of the constraints could be lifted, and the results are shown in Tables 6 and 7, column II. In this calculation the estimated error, $\sigma_{i}$, on the $\mathrm{CH}$ stretching frequencies were lowered from $0.01 \omega_{\mathrm{i}}$ to $0.001 \omega_{\mathrm{i}}$.

In general all values seem "reasonable" and the deviation from the initial force field is not large. It appears, that the force field of the $\mathrm{CH}_{3}$ group is very close to $C_{3 v}$ symmetry. The values of $\Delta f_{r}$ and $\Delta f_{\beta}$ (Table 8) are, if real, opposite in sign relative to the values for other acetyl halides. $^{3}$

In the case of $\mathrm{AcI}$ it seems that the $\mathrm{CH}$ stretching region is fairly free of Fermi resonance (FR), probably because of the unusually low frequencies of the methyl bending modes. The most favourable case for FR in AcI is between $v_{2}$ at $2937 \mathrm{~cm}^{-1}$ and a combination band at 2814 $\mathrm{cm}^{-1}$, more than $100 \mathrm{~cm}^{-1}$ from the fundamental frequency. FR cannot be excluded. However, if $v_{2}$ is significantly affected by FR it should result in a poor refinement for especially that fundamental. From Table 7 is seen that this is not the case. If FR is of minor importance a better refinement might be obtained by changing the methyl group structure.

Iteration sequence III and $I V$. A series of iterations were carried out with small changes in the methyl group structure. It turned out that $f_{r r}$, $\Delta f_{r}$ and $\Delta f_{\beta}$ were sensitive to the value of the HCC angle $(\beta)$. In Table 8 column III the results are shown from an iteration similar to that in column II, but with regular tetrahedral structure of the methyl group. It appears that $\Delta f_{r}$ and $\Delta f_{\beta}$ goes towards zero, and $f_{r r}=0.034$, a value McKean ${ }^{3}$ suggests as preferable. However, the iteration does not yield a better fit of the $\mathrm{CH}$ stretching frequencies. Tilting of the methyl group has no influence either. However, introduction of a difference in the $\mathrm{HCH}$ angles (the $\alpha$ 's) has a striking effect. This has recently been reported by McKean for a far more constrained system. ${ }^{3}$ The fit of the $\mathrm{CH}$ stretching frequencies was improved if $\alpha_{12}=\alpha_{13}$ were increased and $\alpha_{23}$ correspondingly decreased. However, the value of $f_{r r}$ then increased. The frequency data available are not sufficient to give a unique determination of the methyl structure, but the data suggest that corrections to the methyl group structure will have to be made. In Table 8 column IV a calculation is shown which yields both a reasonable value of $f_{r r}$ and an improved fit of the $\mathrm{CH}$ stretching frequencies. However, the result is far from unquestionable.

For clearness the different structures used in the refinements are collected in Table 9.

\section{CONCLUSIONS AND DISCUSSION}

So far a detailed force field has not been made for other acetyl halides, which precludes any direct comparison with the present study. However, the proximity of the initial and the final force field indicates that acetyliodide behaves roughly as expected. The assignment and calculations presented confirm that the force field of the methyl group in AcI has very nearly $C_{3 v}$ symmetry. The calculations also confirm that the fit of the $\mathrm{CH}$ stretching and bending modes are very sensitive to the methyl group structure. Our calculations suggest that the (average) $\mathrm{CCH}$ angles are larger than the previously published value of $108.1^{\circ}$. The data suggest a value close to the regular tetrahedral angle $\left(109.47^{\circ}\right)$. This is in accordance with the empirical correspondence between the $\mathrm{CCH}$ angle and the isolated $\mathrm{CH}$ stretching frequency given by McKean. ${ }^{23}$ However, the data also suggest that the methyl group structure deviates from $C_{3 v}$ symmetry, because the $\alpha$ 's (and perhaps also the $\beta$ 's) are different.

The asymmetry in the methyl group structure may seem in conflict with the near $C_{3 v}$ symmetry of the force field. Our explanation is that in the case of AcI the asymmetry of the methyl group is mainly due to different sterical interactions between the individual $\mathrm{CH}$ bonds and the $\mathrm{COI}$ moiety. The methyl group compensates for this asymmetric interaction by changing the angular symmetry slightly away from $C_{3 v}$ symmetry, without perturbing the force field significantly. Significant changes in the symmetry of both the structure (including the bond lengths) and the force field, as observed in $\mathrm{AcF}^{3,24}$ must be 
caused by a covalent or "through bond" interaction between the unequivalent $\mathrm{CH}$ bonds and the skeleton. Because the final methyl group structure is the resultant of two effects, which may be opposite in direction, there is not necessarily a correlation between the asymmetry in the $\mathrm{CH}$ bond lengths (or the $\mathrm{CH}$ stretching force constants) and the angular symmetry of the methyl group. This conclusion is supported by the experimental data of $\mathrm{McKean}^{3}$ and that obtained from $a b$ initio calculations given by Boggs et al. ${ }^{25}$ Further work on $\mathrm{AcF}$ and $\mathrm{AcCN}$ is in progress in our laboratory.

Acknowledgement. The authors are grateful to Dr. G. O. Sørensen and the Computer Department at the H. C. Ørsted Institute for giving us access to the computer programs used in the force field calculations, and to the Danish Natural Science Research Council for partially financing the infrared equipment.

\section{REFERENCES}

1. Seewan-Albert, H. and Kahovec, L. Acta Phys. Aust. 1 (1948) 354.

2. Ramsey, J. A. and Ladd, J. A. J. Chem. Soc. $B$ (1968) 118.

3. McKean, D. C. and Torto, I. J. Mol. Struct. 81 (1982) 51.

4. Moloney, M. J. and Krisher, L. C. J. Chem. Phys. 45 (1966) 3277.

5. Tsuchiya, S. J. Mol. Struct. 22 (1974) 77.

6. Szwarc, M. and Murawski, J. Trans Faraday Soc. 47 (1951) 269.

7. Hansen, E. L. and Nicolaisen, F. M. Infrared Phys. 19 (1979) 225.

8. Wilson, E. B., Decius, J. C. and Cross, P. C. Molecular Vibrations, McGraw Hill, New York 1955.

9. Ueda, T. and Shimanouchi, T. J. Mol. Spectrosc. 28 (1968) 350.

10. Seth-Paul, W. A. and Dijkstra, G. Spectrochim. Acta Part A 23 (1967) 2861.

11. Seth-Paul, W. A. J. Mol. Struct. 3 (1969) 403.

12. Duncan, J. L. Spectrochim. Acta 20 (1964) 1197.

13. King, W. T. and Crawford, B., Jr. J. Mol. Spectrosc. 5 (1960) 421.

14. Overend, J. In Davies, M., Ed., Infrared Spectroscopy and Molecular Structure, Elsevier, Amsterdam 1963, p. 352.
15. Nicolaisen, F. M. and Hansen, J. S. Mol. Phys. 47 (1982) 1265.

16. Aldous, J. and Mills, I. M. Spectrochim. Acta 18 (1962) 1073.

17. Aldous, J. and Mills, I. M. Spectrochim. Acta 19 (1963) 1567.

18. Bjørklund, S., Augdahl, E., Christensen, D. H. and Sørensen, G. O. Spectrochim. Acta Part A 32 (1976) 1021.

19. Suzuki, M., Yamada, K. and Takami, M. J. Mol. Spectrosc. 88 (1981) 207.

20. Davies, D. W. and Gerry, M. C. L. J. Mol. Spectrosc. 97 (1983) 117.

21. Hollenstein, H. and Günthard, H. H. Spectrochim. Acta Part A 27 (1971) 2027.

22. Duncan, J. L., McKean, D. C., Mackenzie, M. W. and Peña, J. J. Mol. Spectrosc. 76 (1979) 55.

23. McKean, D. C. J. Mol. Spectrosc. 34 (1976) 181.

24. Pierce, L. and Krisher, L. C. J. Chem. Phys. 31 (1959) 875.

25. Flood, E., Pulay, P. and Boggs, J. E. J. Am. Chem. Soc. 99 (1977) 5570.

Received November 7, 1983. 